\title{
Development and validation of a tool for assessing understanding of brain death
}

\begin{abstract}
Background-Death by neurological criteria is often misunderstood by laypersons even though they make decisions about withdrawal of care and organ donation. No validated questionnaire for determining laypersons' understanding of brain death exists. Such a tool could be useful in clinical, educational, and research settings. Methods-Brain death experts and a focus group of laypersons were used to develop a 5-item questionnaire with face validity. The questionnaire explores 3 concepts: apnea, irreversibility, and differentiation between cardiac death, brain death, and persistent vegetative state. The questionnaire was administered to separate groups of laypersons and experts and was readministered 7 to 10 days later. Test-retest reliability for individual items and overall score was measured by using Spearman rank correlation. Internal consistency of the questionnaire was measured by using Cronbach $\alpha$. Utility of the questionnaire in discriminating between scores of laypersons and experts was evaluated by using a $t$ test.

Results-Twelve laypersons and 13 experts participated. The test-retest correlation was significant for all questions (Spearman $\rho$ range, 0.43-0.94) and raw score (Spearman $\rho=0.91, P<.001$ ). Internal consistency was fair (Cronbach $\alpha=0.64$ ). The questionnaire enabled discrimination of laypersons from experts, with mean (SD) raw scores of $3.0(1.1)$ vs $4.8(0.6)$, respectively $(t$ test, $P<.001)$. Removal of 1 item improved internal consistency (Cronbach $\alpha=0.70$ ), but with a corresponding decrease in discriminatory ability.

Conclusions-This simple 5-item questionnaire for evaluating understanding of brain death has test-retest reliability, internal consistency, and can be used to discriminate between persons who do and do not understand brain death. (Progress in Transplantation. 2009;19:272-276)
\end{abstract}

\author{
Isaac Tawil, MD, Jonathan \\ Marinaro, MD, Lawrence $\mathbf{H}$. \\ Brown, MPH\&TM, EMT-P \\ University of New Mexico Health \\ Sciences Center, Albuquerque
}

Corresponding author: Isaac Tawil, MD, University of New Mexico Health Sciences Center, Department of Surgery/Emergency Medicine, MSC10-5610, 1 University of New Mexico, Albuquerque, NM 87131 (e-mail: itawil@salud.unm.edu)

To purchase electronic or print reprints, contact:

The InnoVision Group

101 Columbia, Aliso Viejo, CA 92656

Phone (800) 809-2273 (ext 532) or

(949) 448-7370 (ext 532)

Fax (949) 362-2049

E-mail reprints@aacn.org

\begin{abstract}
$T^{2}$ he concept of brain death is difficult for laypersons to understand.' Previous studies have shown that one-third of relatives of patients diagnosed with brain death did not understand that death had occurred. ${ }^{2}$ This lack of understanding may compound the family members' grief and complicate decision making with respect to organ donation. In one study, ${ }^{3}$ next of kin who decided against organ donation had far less understanding of brain death than did next of kin who decided in favor of donation. Experts agree that donation refusal rates are linked to the lack of understanding of brain death. In a study ${ }^{4}$ of family members of potential organ donors in Ohio and Pennsylvania, researchers found that $28.3 \%$ of the 403 participants could provide an accurate definition for brain death, but only $15.8 \%$ of the subjects equated brain death with death. Similarly, in a study from Brazil, researchers reported that $80 \%$ of laypersons would authorize organ donation from a dead relative who had previously expressed a willingness to
\end{abstract}

donate organs. But, when asked if they would authorize donation from a "brain-dead" relative, the percentage decreased to $63 \% .^{5}$ In a recent review of the topic, Long et $\mathrm{al}^{6}$ concluded that a sustained increase in the number of organs available for transplantation may never be achieved until bereaved family members of their deceased relative have a better understanding of brain death.

Currently, no validated tool is available for objectively evaluating understanding of brain death; such a tool could be useful in clinical practice, education, and research. Therefore, we undertook this project to develop and validate a tool for assessing understanding of brain death.

\section{Methods}

This study was approved by the Human Research Review Committee at the University of New Mexico Health Sciences Center. 
Table 1 First-iteration questionnaire

1. Can someone who is brain dead breathe without the support of a breathing machine?

2. Can someone who is brain dead ever wake up (recover)?

3. Do you remember the case of Terri Schiavo? ${ }^{a}$

4. Does being brain dead mean being like Terri Schiavo?

a This question was not scored, but rather was used to determine whether the answer to question 4 should be scored (if question 3 answered yes) or omitted (if question 3 answered no).

\section{Questionnaire Development}

Phase I. A panel of experts on brain death and bereavement spanning the disciplines of intensive care medicine, neurosurgery, trauma surgery, and psychiatry was convened to advise the development of the tool. They identified 3 constructs such a tool should measure: (1) that brain-dead patients are completely apneic and require mechanical ventilation, (2) that brain death is irreversible, and (3) that brain death is distinct from a "persistent vegetative state." They also commented that, given the clinical circumstances in which such a tool might be used, it must be simple and brief.

We then consulted with a small focus group of 3 laypersons to explore these concepts and how best to ask other laypeople about them. The result was the 4-item questionnaire, addressing the 3 constructs identified by the expert panel (Table 1). This initial questionnaire was then evaluated for test-retest reliability and internal consistency. The questionnaire was distributed to a convenience sample consisting of a class of 12 undergraduate paramedic students and then readministered 10 days later. Test-retest reliability among the 10 students completing the questionnaire on both days was measured by using Spearman rank correlation and internal consistency was measured with Cronbach $\alpha$ (Table 2).

Phase II. Because of the low internal consistency and poor test-retest reliability of one of the questions in this initial questionnaire, we again consulted the expert panel and the layperson focus group to revise the questionnaire. The questions relating to Ms Schiavo were removed, and 3 new questions-one addressing core reflexes, one differentiating between cardiac and neurological death, and one that explicitly requires respondents to differentiate between brain death and a persistent vegetative state-were crafted. The revised questionnaire is shown in Table 3. We then evaluated this newer version of the questionnaire for test-retest reliability and internal consistency, as well as its utility in discriminating between laypersons' and experts' understanding of brain death.
Table 2 First-iteration test-retest correlation and internal consistency

\begin{tabular}{lcc}
\hline Question No. & Spearman $\rho$ & $P$ \\
\hline 1 & 1.00 & $<.001$ \\
2 & 0.95 & $<.001$ \\
3 & NA & NA \\
4 & 0.62 & .133 \\
Raw score & 1.00 & $<.001$ \\
Composite & 0.83 & .003 \\
Cronbach $\alpha=0.04$ & & \\
Abbreviation: NA, not applicable. & \\
\hline
\end{tabular}

\section{Questionnaire Evaluation}

The final questionnaire, which is written at a 6thto 7 th-grade reading level (Flesh-Kincaid grade level assessment), was administered to nonclinical administrative support personnel working in an academic medical center (laypersons), and to physicians, physician extenders, and nurses who work in neurosurgical and other clinical departments related to organ procurement at the same academic institution (experts). The questionnaire was readministered to the same individuals 7 to 10 days later. A unique identifier was assigned to each participant so that the research team was blinded to individual identities.

Test-retest correlation was evaluated by using Spearman rank correlation, with $P$ less than .05 considered statistically significant. Test-retest correlation was evaluated for individual questionnaire items as well as raw score (sum of correct responses) and a composite score for complete understanding (ie, no incorrect responses). Internal consistency of the questionnaire items was measured by conducting reliability analysis and calculating Cronbach $\alpha$. The utility of the questionnaire in discriminating between persons who do and do not understand brain death was measured by comparing the scores of the laypersons with the scores of the experts by using a $t$ test, with $P$ less than .05 considered statistically significant. All data management and analyses were conducted by using SPSS version 16.0 (SPSS, Inc, Chicago, Illinois).

Table 3 Final questionnaire ${ }^{a}$

1. Can someone who is brain dead breathe without the support of a breathing machine?

2. Can someone who is brain dead ever wake up (recover)?

3. Will someone who is brain dead react (grimace, move away, or blink) if someone touches their eyeball?

4. Can a person be brain dead even if their heart is still beating?

5 . Is brain death different from a coma or a vegetative state?

a Participants answer Yes, No, or Unsure to each question and are scored 1 point for each correct answer. 
Table 4 Final questionnaire test-retest responses ${ }^{a}$

\begin{tabular}{|c|c|c|c|c|c|c|}
\hline \multirow[b]{2}{*}{ Question No. } & \multicolumn{2}{|c|}{ Overall $(n=25)$} & \multicolumn{2}{|c|}{ Laypersons $(n=12)$} & \multicolumn{2}{|c|}{ Experts $(n=13)$} \\
\hline & Test & Retest & Test & Retest & Test & Retest \\
\hline$\overline{1}$ & 17 & 18 & 6 & 7 & 11 & 11 \\
\hline 2 & 20 & 21 & 7 & 8 & 13 & 13 \\
\hline 3 & 16 & 17 & 4 & 5 & 12 & 12 \\
\hline 4 & 22 & 24 & 9 & 11 & 13 & 13 \\
\hline 5 & 23 & 23 & 10 & 10 & 13 & 13 \\
\hline Raw score, median (range) & $4(1-5)$ & $5(2-5)$ & $3(2-5)$ & $3(2-5)$ & $5(3-5)$ & $5(3-5)$ \\
\hline Composite (No. with 0 incorrect) & 12 & 14 & 1 & 3 & 11 & 11 \\
\hline
\end{tabular}

\section{Results}

\section{Test-Retest Validity}

Twelve nonclinical administrative personnel and 13 clinician experts completed both the test and retest questionnaires. Responses for each item are shown in Table 4. Overall, correlation was significant between the test and retest responses for all questions. Testretest correlation was weaker, as expected, among laypersons. Correlation was also significant in the overall scoring of the questionnaire, both in terms of raw score (cumulative number of items correct) and composite understanding (no incorrect responses vs some incorrect responses); these correlations were strong in the data for laypersons, experts, and overall. The test-retest correlation data are shown in Table 5.

\section{Internal Consistency}

Reliability analysis of the 5 items contained in the second-iteration questionnaire revealed an overall Cronbach $\alpha$ of 0.64 . Subsequent factor analysis using a component matrix revealed that questions $1,2,4$, and 5 loaded on the same component, whereas question 3 (about core reflexes) loaded more strongly on a separate component. This effect was almost entirely within the layperson subgroup. In the layperson subgroup, the 5-item questionnaire had a Cronbach $\alpha$ of 0.62 , which improved to 0.72 when question 3 was removed. In the combined data from laypersons and experts, removal of question 3 yields a Cronbach $\alpha$ of 0.70 , which is the threshold most commonly used to demonstrate internal consistency. (Without any variance in the expert responses, calculation of Cronbach $\alpha$ for question 3 for this subgroup alone is meaningless.)

\section{Discriminatory Ability}

The raw test scores for the laypersons (mean, 3.0; SD, 1.1) differed significantly ( $t$ test, $P<.001$ ) from the scores for the expert respondents (mean, $4.8 ; \mathrm{SD}$, 0.6 ). When question 3 is removed from the analysis, the difference is smaller (2.7 [1.2] vs $3.9[0.4])$, but still statistically significant ( $t$ test, $P=.005$ ).

\section{Discussion}

This brief, simple questionnaire for measuring understanding of brain death has good internal consistency, significant test-retest validity, and utility for discriminating between persons with a layperson's versus an expert's understanding of brain death.

Although previous researchers have evaluated understanding of brain death, ${ }^{4,6}$ those authors have not reported efforts to validate their assessment tools. Siminoff et $\mathrm{al}^{4}$ asked family members of potential organ donors to define brain death, to describe the braindeath testing process, and to describe how and when (or if) they came to accept that their family member was indeed dead. Franz et al, ${ }^{6}$ in their analysis of 164 family members of potential organ donors, included questions similar to those in our final questionnaire differentiating between brain death, cardiac death, and persistent vegetative states, as well as questions addressing the irreversible nature of brain death. Our study now provides evidence of the internal consistency, test-retest reliability, and discriminatory utility of these questions, along with the 2 additional questions relating to apnea and absence of core reflexes.

A reliable and valid tool for assessing understanding of brain death has many potential uses. Such a tool could be useful as an assessment tool in clinical situations to determine whether patients' family members and other loved ones actually understand brain death before they make end-of-life decisions about such things as withdrawal of life support or organ donation..$^{1-4}$ In these highly emotional circumstances, it is unlikely that the questionnaire would be administered in written form, but clinicians and counselors working with family members could incorporate these 5 constructs into their discussions to evaluate understanding. The questionnaire could also be used to guide 
Table 5 Final questionnaire test-retest correlation

\begin{tabular}{|c|c|c|c|c|c|c|}
\hline \multirow[b]{2}{*}{ Question No. } & \multicolumn{2}{|c|}{ Overall } & \multicolumn{2}{|c|}{ Laypersons } & \multicolumn{2}{|c|}{ Experts } \\
\hline & Spearman $\rho$ & $P$ & Spearman $\rho$ & $P$ & Spearman $\rho$ & $P$ \\
\hline 9 & 0.94 & $<.001$ & 0.90 & $<.001$ & 1.00 & $<.001$ \\
\hline 2 & 0.45 & .025 & 0.45 & .144 & ${ }^{*}$ & \\
\hline 3 & 0.72 & $<.001$ & 0.69 & .013 & 1.00 & $<.001$ \\
\hline 4 & 0.55 & .004 & 0.52 & .082 & $\mathrm{a}$ & \\
\hline 5 & 0.43 & .030 & 0.35 & .267 & $a$ & \\
\hline Raw score & 0.91 & $<.001$ & 0.75 & .003 & 1.00 & $<.001$ \\
\hline Composite & 0.85 & $<.001$ & 0.52 & .082 & 1.00 & $<.001$ \\
\hline
\end{tabular}

presentations and assess understanding in communitybased programs designed to increase awareness about organ donation.

The questionnaire could also be useful in medical education settings, particularly with regard to assessing physicians' and other health care providers' understanding of brain death. Although physicians and other medical personnel would be expected to have a more sophisticated understanding of brain death than laypersons have, such is not always the case. Youngner et $\mathrm{al}^{7}$ presented health care providers involved in the care of brain-dead patients with 1 factual question regarding the definition of brain death and 2 scenarios in which to apply that definition. Only $63 \%$ correctly identified brain death as "irreversible loss of all brain function," and only $35 \%$ were able to provide both a correct definition for brain death and an appropriate determination for both scenarios. In a more recent study using a similar factual question and a single scenario to assess understanding of brain death among pediatricians, Harrison and Botkin ${ }^{8}$ reported that only $36 \%$ of residents $(n=42)$ correctly defined brain death and only $38 \%$ of those 42 residents correctly interpreted the scenario; attending pediatricians performed slightly better, with $39 \%(n=44)$ providing an accurate definition and $64 \%$ of those 44 pediatricians appropriately interpreting the scenario. Although our survey is simplistic, it addresses the salient concepts of complete loss of brain function (as evidenced by apnea and loss of core reflexes), differentiation from cardiac death as well as from a persistent vegetative state, and irreversibility.

Finally, the questionnaire has a number of potential research applications, including potential use in studies evaluating the association (if any) between understanding of brain death and organ donation. ${ }^{1-4}$ In fact, we are now using the tool in an ongoing study of family presence during the brain-death examination.

\section{Limitations and Future Studies}

One limitation of this questionnaire is that it has been derived and validated in the same setting. Although different populations were used for the derivation and validation processes, it is possible that the questionnaire might perform differently in other settings. Also, although we have demonstrated an ability to measure understanding of brain death, we have not measured whether that understanding, or different levels of understanding, is indeed associated with decisions about withdrawal of life support or organ donation. The importance of good communication between clinicians and family members, and the impact of such communication on understanding, cannot be overemphasized. Future research is necessary to validate this questionnaire in other settings and to explore the associations, if any, between understanding of brain death and end-of-life decision making.

Another limitation of this questionnaire is the relative weakness of question 3 . Although question 3 detracts from the internal consistency of the questionnaire, this problem is entirely due to responses from the layperson subgroup. However, although the questionnaire can be used to differentiate between novices and experts without the inclusion of question 3 , inclusion of that question does increase the discriminatory value of the questionnaire. Therefore, we believe that question 3 should remain a part of the questionnaire unless and until further evaluation demonstrates that that question is not useful.

\section{Conclusion}

This simple 5-item questionnaire for evaluating understanding of brain death has test-retest reliability, internal consistency, and utility in discriminating between persons who do and do not understand brain death. The questionnaire has potential applications in clinical, educational, and research settings. 


\section{Tawil et al}

\section{Acknowledgment}

This work was presented at the annual meeting of the Neurocritical Care Society, Miami Beach, Florida, October 2008 .

\section{Financial Disclosures}

None reported.

\section{References}

I. Ormrod JA, Ryder T, Chadwick RJ, Bonner SM. Experiences of families when a relative is diagnosed brain stem dead: understanding of death, observation of brain stem death testing and attitudes to organ donation. Anaesthesia. $2005 ; 60(10)$ : 1002-1008.

2. Pearson IY, Bazeley P, Spencer-Plane T, Chapman JR, Robertson P. A survey of families of brain dead patients: their experiences, attitudes to organ donation and transplantation.
Anesth Intensive Care. 1995;23(1):88-95.

3. Franz HG, DeJong W, Wolfe SM, et al. Explaining brain death: a critical feature of the organ donation process. $J$ Transplant Coord. 1997;7(1):14-21.

4. Siminoff LA, Mercer MB, Arnold R. Families' understanding of brain death. Prog Transplant. 2003;13(3):218-224.

5. Tessmer CS, da Silva AR, Barcellos FC, Araujo CL, da Costa JD, Bohlke M. Do people accept brain death as death? A study in Brazil. Prog Transplant. 2007;17(1):63-67.

6. Long T, Sque M, Addington-Hall J. What does a diagnosis of brain death mean to family members approached about organ donation? A review of the literature. Prog Transplant. 2008; 18(2):118-126.

7. Youngner SJ, Landefeld CS, Coulton CJ, Juknialis BW, Leary M. 'Brain death' and organ retrieval: a cross-sectional survey of knowledge and concepts among health professionals. JAMA. 1989;261(15):2205-2210.

8. Harrison AM, Botkin JR. Can pediatricians define and apply the concept of brain death? Pediatrics. 1999;103(6):e82. 